Int. J. Dev. Biol. 55: 917-921

doi: $10.1387 / \mathrm{ijdb} .113288 \mathrm{sh}$

\title{
Xler2 is required for convergent extension movements during Xenopus development
}

\author{
SUNG-KOOK HONG ${ }^{1,2}$, KOSUKE TANEGASHIMA ${ }^{1,3}$ and IGOR B. DAWID ${ }^{*, 1}$ \\ ${ }^{1}$ Laboratory of Molecular Genetics, Program in Genomics of Differentiation, Eunice Kennedy Shriver National \\ Institute of Child and Human Development, NIH, USA, ${ }^{2}$ Molecular Genetics Branch, National Human Genome \\ Research Institute, NIH, Bethesda, MD, USA and ${ }^{3}$ Tokyo Metropolitan Institute of Medical Science, Tokyo, Japan
}

\begin{abstract}
Immediate early response 2 (ler2) is a downstream target of fibroblast growth factor (FGF) signaling. In zebrafish, ler2 is involved in left-right asymmetry establishment and in convergent extension movements. We isolated the Xenopus ier2 gene based on sequence similarity searches using multiple vertebrate species. Xenopus ler2 has high homology in the $\mathrm{N}$-terminal region to other vertebrate ler2 proteins, and Xier2 transcripts were observed from oocytes through larval stages. Except for the maternal expression of xier2, the expression of this gene in the marginal region at gastrulation and in somites and the notochord at later stages is similar to the expression pattern of zebrafish ier2. Xler2 knockdown using antisense morpholinos resulted in defects of convergent extension leading to severe neural tube defects; overexpression of ler2 showed similar, albeit milder phenotypes. Assays in animal cap explants likewise showed inhibition of elongation after blocking Xler2 expression. These results indicate that Xenopus ler2 is essential for the execution of convergent extension movements during early Xenopus development.
\end{abstract}

KEY WORDS: XIer2, Xenopus, convergent extension

\section{Introduction}

Immediate early response 2 (ler2) has been identified as a growth factor-inducible protein, and the signals involved in its expression have been described (Charles et al., 1990; Latinkic and Lau, 1994), but the functions of ler2 in the mouse embryo have not been reported to date. Recently we have isolated zebrafish ler2 as a downstream target of FGF signaling and described its role in left-right asymmetry patterning in this animal (Hong and Dawid, 2009). In addition to affecting left-right asymmetry, knockdown of zebrafish ler2 also results in defects in convergent extension movements from late gastrula through early segmentation stages. Convergent extension movements are critical in both Xenopus and zebrafish for the elongation of involuting tissue and for establishing the anterior-posterior body axis of the embryo (Keller et al., 2000; Wallingford et al., 2002; Heisenberg etal., 2000; Sepich et al., 2000).

To investigate whether the functions of ler2 are conserved among different vertebrate species we examined the role of this protein in gastrulation and post-gastrulation development of a tetrapod. Here we report the isolation of the two Xenopus ier2 genes and their expression during development. Using an antisense morpholino (MO) to attenuate ler2 expression, we probed the role of ler2 protein in the embryo and in animal explants. Our observations indicate that Xler2 has a critical role in convergent extension movements in the early development of this animal.

\section{Results}

\section{Isolation of Xenopus laevis ier2}

To examine the roles of Xenopus ler2 during embryonic development we identified Xenopus tropicalis ler2 by searching its genome using the highly conserved $\mathrm{N}$-terminal region of other vertebrate ler2 sequences. This search led to a contig, Scaffold_649, in the Ensembl genome browser, which provided us with full-length sequence information for $X$. tropicalis ier2. We designed PCR primers based on $X$. tropicalis sequence information, allowing us to isolate $X$. laevis ier2 cDNA. As is common, $X$. laevis contains two pseu-

Abbreviations used in this paper: fgf, fibroblast growth factor; Ier, immediate early response gene; mo, morpholino.

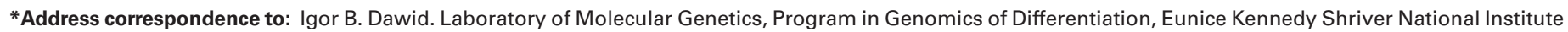
of Child Health and Human Development, Bethesda MD 20892, USA. e-mail: idawid@nih.gov
}

Supplementary Material (one figure) for this paper is available at: http://dx.doi.org/10.1387/ijdb.113288sh 
A

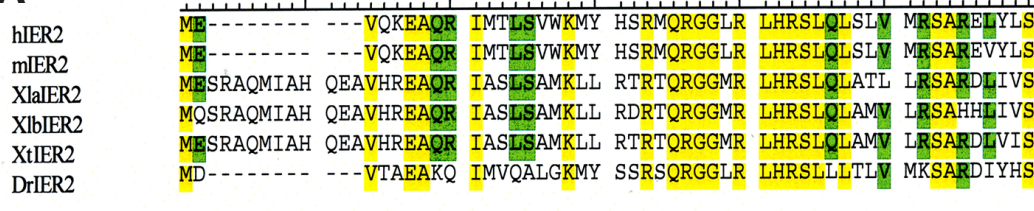
hIER2 mIER2

XlaIER2

XIbIER2

XtER2

DrIER2

hIER2

mIER2

XlaIER2

XIbIER2

XtIER2

DrIER2

hIER2

mIER2

XlaIER2

XIbIER2

XtIER2

DrIER2

hIER2

mIER2

XlaIER2

XlbIER2

XtIER2

B

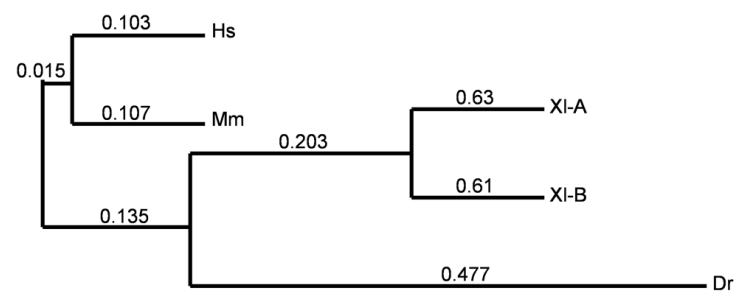

A

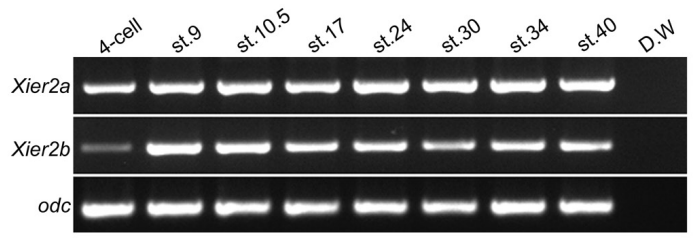

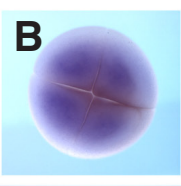
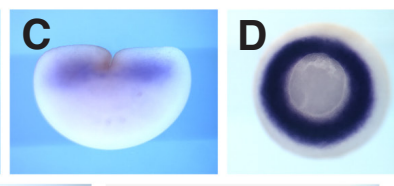

E
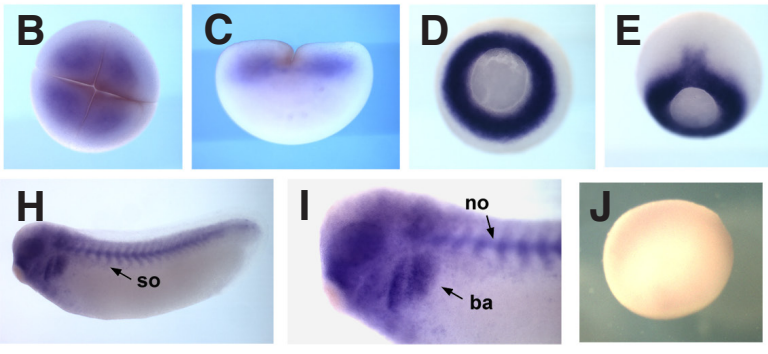

110

AKVEALEPEV SLPAALPSDP RLHPRREAES TAETATPDGE HPFPEPMDTQ EAPT-AEETS AKVEAHQPEF R-PSRRALDP RLHPPREDEV AVEVASPEAV QP-PGPMDTQ EEVLRVQETP T--ILPQEE R-1.-. -

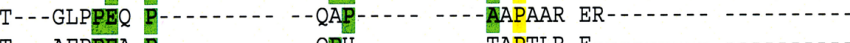
ARLMSEKSGQ S---VTEEC TSHTQEPMDT SSSTATPLRE TSGQSSEDGQ RSGLEGHPHP

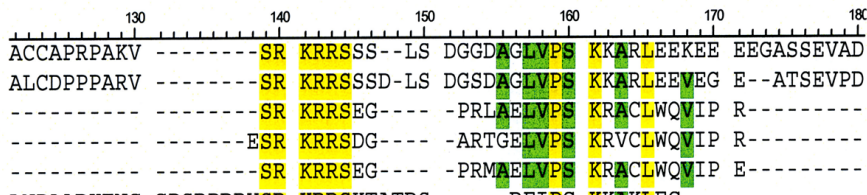

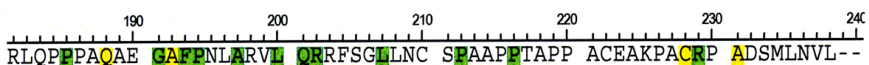
RLQLPPAQSE GAFPNLARVL QRRFSSFLNC GPAVPPPTPP TCEAKPACRP ADNMLNVL--.--PQPQG- GAFPGMAEIL QR----EIGS VPQPQPLG-- --.--CRE AFAVPGFH--.--PQPQG- GAFPGMAEIL QR----ELGS VPRVQPVR-- -.----CRE ALGLPGFH----PQPQG - GAFPGMAEVE QR----LIGS IPQAQPLG-- -..--CRA AMAVPGFH-AEVRGVLQNS SAN-

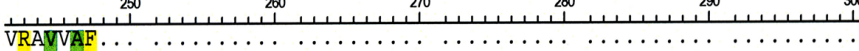

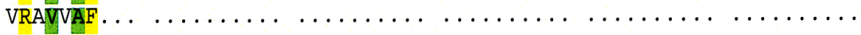

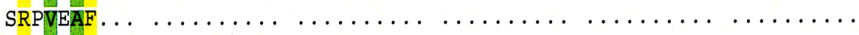

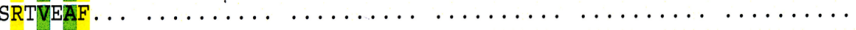

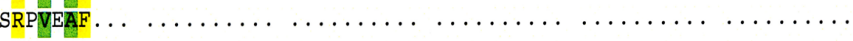

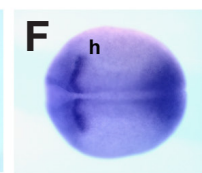

G

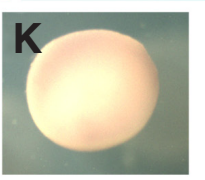

Fig. 1. Alignment of vertebrate ler2 sequences. (A) Comparison of amino acid sequences of vertebrate ler 2 proteins including human ler2 (h) (NM_004907), mouse ler2 (m) (NM_010499), X. laevis ler2a (Xla) (GQ120520), X. laevis ler2b (XIb) (GQ120521), $X$. tropicalis $(X t)$ (predicted sequence based on Scaffold_649 of genomic sequence), and zebrafish ler2 (Dr) (NM_001142583). Conserved sequences are highlighted in yellow and green. (B) Phylogenetic tree of vertebrate ler2 proteins.

doalleles of ier2, which share $87 \%$ amino acid sequence identity. The sequences have been deposited in the public data base as xier2a (GenBank accession \# GQ120520) and xier2b(GenBank accession\#GQ120521). Alignment of the corresponding protein sequences with ler2 from other vertebrate species shows that this protein displays substantial conservation among several vertebrates (Fig. $1 \mathrm{~A})$. The evolutionary relationship between vertebrate ler2 proteins is shown in Fig. 1B.

\section{Expression of xier2 during development}

To examine expression of xier2, we performed semiquantitative RT-PCR analysis and whole mount in situ hybridization using both of xier2a and xier2b. Xier2 transcripts of both genes were detected in maternal RNA and during embryogenesis through tadpole stages (Fig. 2A). The spatial expression of $x$ ier2bwas visualized using whole mount in situ hybridization during development of Xenopus. Transcripts were observed before gastrulation in the animal region (Fig $2 \mathrm{~B}, \mathrm{C}$ ), and in the marginal region during gastrulation (Fig. $2 \mathrm{D}, \mathrm{E})$. At st. 13 xier2b transcripts were detected in the hindbrain and paraxial mesoderm (Fig. 2F). These domains of expression are maintained during later stages, and in addition branchial arches, somites, and the notochord showed expression during tailbud to tadpole stages (Fig. 2 G-I). Sense strand controls showed no staining at any stage tested (Fig. $2 \mathrm{~J}$-L). The xier2a expression pattern is similar to the pattern of $x i e r 2 b$ shown in Fig. 2 (data not shown). Furthermore, in the marginal region, notochord and arch primordia, the expression of $x i e r 2 b$ was similar to the pattern seen for zebrafish ier2 (Hong and Dawid, 2009),

Fig. 2. Expression patterns of xier2. (A) $R T-P C R$ analysis of Xenopus ier2a and $b$ genes from early cleavage to st 40 . Odc was used as control. (B-I) Whole mount in situ hybridization of xier2b, using antisense strand probe; (J-L) sense strand used as control. $(\mathbf{B}, \mathbf{C})$ Maternal expression is seen in whole mount and transverse section of two-cell stage embryo (C). (D,E) Restricted expression of xier $2 \mathrm{~b}$ in the marginal zone and involuting axial mesoderm during gastrulation at stage 11. (F,G) Hindbrain and somite expression of xier2 at st $13(\mathbf{F})$ and st 25 (G). $(\mathbf{H}, \mathbf{I})$ Expression of xier2b in branchial arches, notochord, and somites at stage 30; (I) is a magnified view of the head region. ba, branchial arches; $h$, hindbrain; no, notochord, so, somites. (J-L) Embryos at st 11 (J,K) and st 29/30 (L) hybridized with sense strand as controls. 
suggesting similar functions for ler2 during embryonic development of Xenopus and zebrafish.

\section{Convergent extension defects result from knockdown and overexpression of Xler2}

To investigate the function of Xler2 in Xenopus development, anti-sense Xler2 MOs were designed to deplete endogenous Xler2 by targeting the translation start site. We injected Xier2 MO into 4-cell stage embryos into each of the two dorsal blastomeres and analyzed the resulting phenotype. Injection of Xler2b MO at $40 \mathrm{ng}$ per embryo resulted in a severe phenotype indicating inhibition of convergent extension movements, leading to a shortened axis and frequently an open neural tube, whereas injection of 60ng control $\mathrm{MO}$ had no effect on development (Fig. 3 A,D,H). Similar levels of an MO targeting Xler2a had a much weaker effect on development, and therefore we restricted our attention on the Xler2b MO. Overexpression of ler2 by injection of synthetic mRNA also led to phenotypes that were less severe than those of $\mathrm{MO}$ injection, and
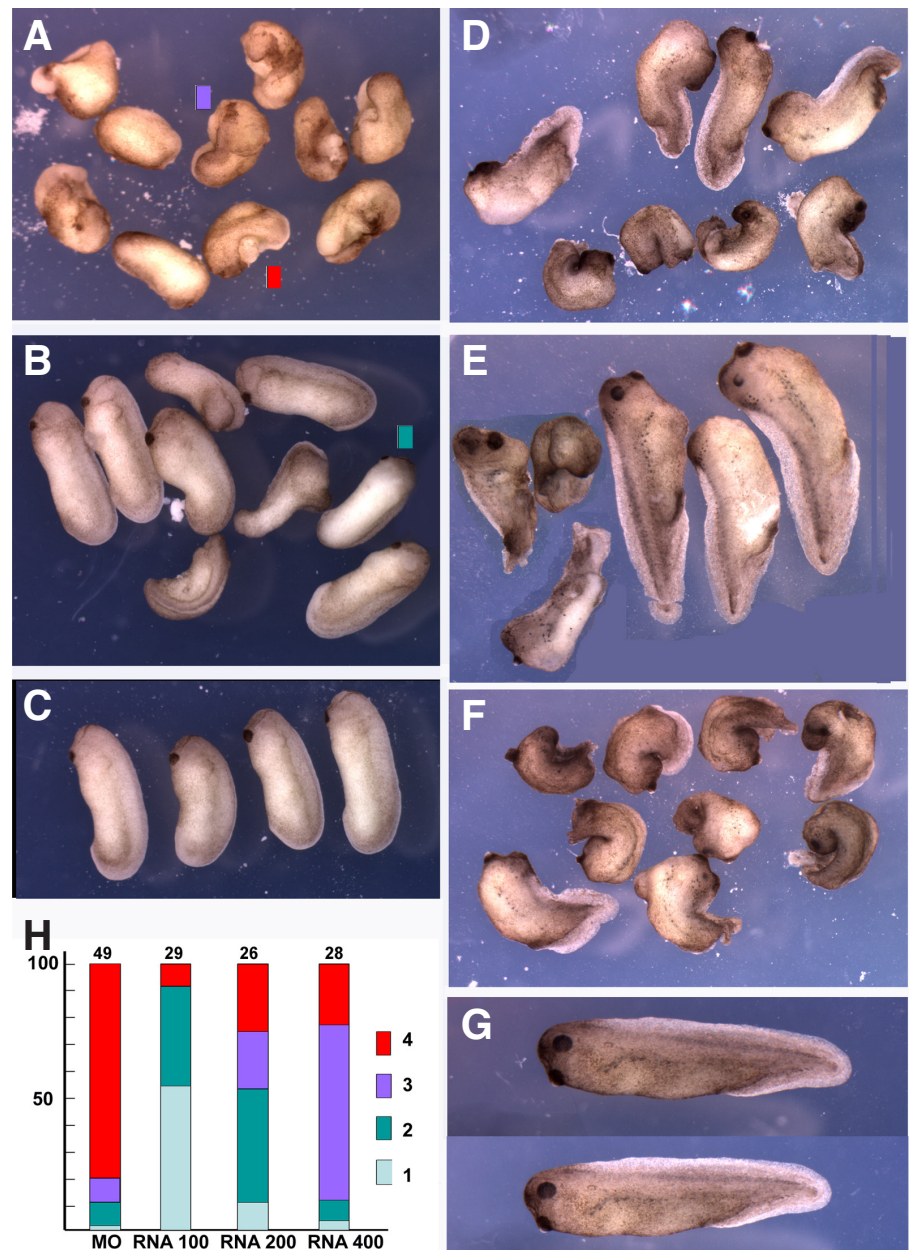

were dosage dependent (Fig. 3 B,E,F,H). Rescue of whole-embryo knock-down phenotype was not achieved, possibly because both $\mathrm{MO}$ and mRNA generate a phenotype and achieving a true balance proved impractical. However, the specificity of the MO is indicated by the rescue that could be achieved in animal explant experiments, as shown below.

To further study the effect of this MO we used animal caps that are induced to elongate by treatment with activin; induced animal caps undergo cell movements that are appropriate model systems for convergent extension in the embryo (Tada and Smith, 2000; Wallingford et al., 2000). Untreated animal caps fail to elongate (Fig. 4A), while activin-treated explants elongate (Fig. 4B). This elongation was strongly inhibited by knockdown of Xler2b (Fig. 4C) and importantly, was rescued to a large extent by the co-injection of $100 \mathrm{pg}$ of $x i e r 2 b$ mRNA (Fig. 4D). The respective phenotypes were $100 \%$ penetrant in these treatments (Fig. 4). These data support the view that ler2 function is required for convergent extension movements in the Xenopus gastrula embryo.

The phenotypes observed after Xier2 knock-down were not due to inhibition of mesoderm induction. Several markers of mesoderm differentiation including $x b r a, b m p 4$, and $f z b$, as well as the organizer marker $g s c$ and the neural marker sox 2 were unaffected in animal caps by injection of Xier2 MO (Supplementary Fig. S1A). Likewise, the paraxial mesoderm marker myoD was expressed in $\mathrm{MO}$ as well as RNA injected embryos, albeit in distorted shape (Supplementary Fig. S1 B-D). These observations support the view that Xier2 has a role in convergence and extension movements in the embryo.

\section{Discussion}

In this study we report the isolation of Xenopus laevis ier2cDNA, and a comparison of the amino acid sequences of ler2 proteins
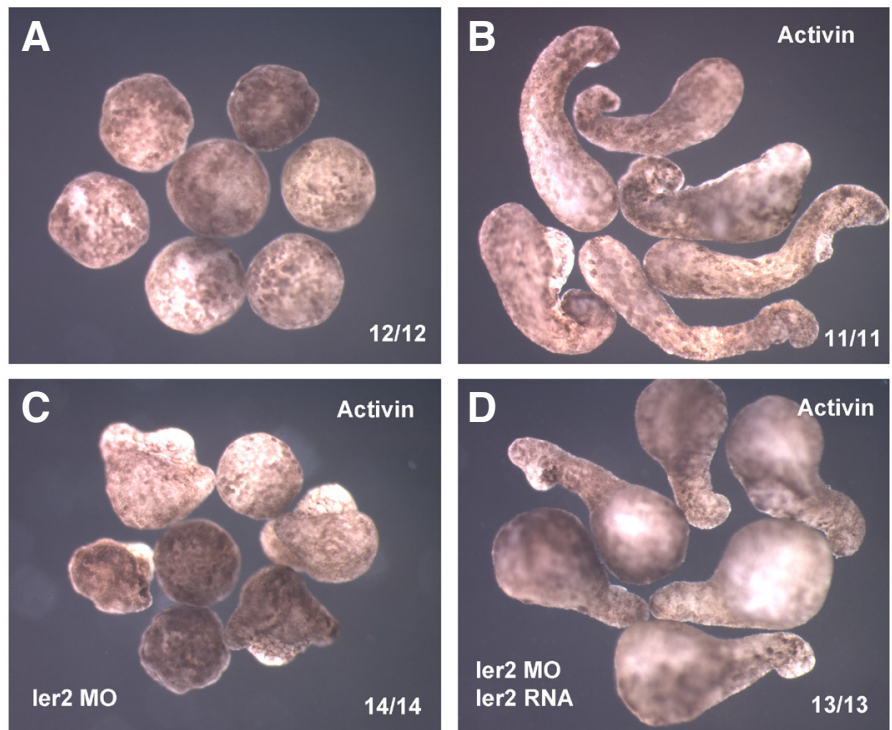

Fig. 3 (Left). Phenotypes generated by ler2 knock-down and overexpression. (A-C) stages 25-27; (D-G) stage 35/36. (A,D) ler2 MO, 40 ng; (B) ier2 RNA, 100 pg; (E) ier2 RNA, 200 pg; (F) ier2 RNA, 400 pg; (C,G) uninjected. (H) quantification of phenotypes: 1, normal; 2, elongated but abnormal; 3 , very short axis with closed blastopore; 4, very short axis with open blastopore. Colored rectangles are placed next to embryos illustrating each phenotype in $(A, B)$. Total number of embryos scored for each injection is shown on top of the histogram, based on two independent experiments.

Fig. 4 (Right). Convergent extension defects in animal caps caused by knockdown of Xler2. Control animal caps round up (A), but elongate after addition of activin (B). Elongation is inhibited by ler2 knockdown (C), and is rescued by co-injection of xier2 mRNA (D). The number of animal caps showing the phenotype portrayed and the number of caps tested is listed in each panel. The data are based on two independent experiments. 
in several vertebrates. As observed previously, vertebrate ler2 proteins have highly conserved $\mathrm{N}$-terminal sequences while the remaining region shows considerable sequence variation (Hong and Dawid, 2009), and this conclusion holds true for the homologs in $X$. laevis and $X$. tropicalis (Fig. 1). The expression pattern of the ier2 gene in Xenopus again shows considerable similarity to the pattern previously observed in zebrafish (Hong and Dawid, 2009). In zebrafish, zygotic expression of ier2 is restricted to the marginal region and developing notochord during gastrulation. At later stages the expression pattern is more dynamic, including the brain, portions of the branchial arches and blood vessels. Many of the expression domains of Xenopus ier2 were the same as those in the zebrafish during early development, except that maternal expression was observed for Xenopus ier2 but not for zebrafish. The similar expression in the marginal zone of both species during gastrulation is of interest because of the defects in convergent extension movements that result in both zebrafish (Hong and Dawid, 2009) and Xenopus embryos as a consequence of the knockdown of ler2. Convergent extension prominently involves the marginal region and the developing notochord, and dorsal marginal explants spontaneously undergo convergent extension movements. These movements lead to the elaboration of the anterior-posterior axis in the embryo.

How does ler2 function in its role in convergent extension? We have previously shown in zebrafish that ier2 is a target gene of Fgf signaling, and is critical in the transmission of the Fgf signal during formation of Kupffer's vesicle, and ultimately in the establishment of left-right asymmetry (Hong and Dawid, 2009). It is possible that ler2 is a more general downstream effector of the FGF pathway, affecting processes beyond left-right asymmetry. Such a hypothesis might well account for the requirement for ler2 in convergent extension as Fgf signaling is well known as an important regulator of this process. The role of Fgf in this context appears to be mediated by different molecules that do not appear to constitute a linear pathway. Brachyury, a well-known Fgf target gene, is required for gastrulation movements in addition to its various roles in mesoderm differentiation and determination of caudal tissues (Conlon and Smith, 1999). However, we find that ler2 knock-down does not inhibit $x b r a$ expression in activin treated animal caps (Supplementary Fig. S1). Some of the effects of Fgf in convergent extension are regulated by the inhibitor Sprouty2 which appears to affect movements differentially from mesoderm differentiation and gene expression (Nutt et al., 2001). More surprising is the observation that $X n r 3$, related in sequence to the Tgf- $\beta$ family, affects convergent extension through the Fgf receptor 1 (Yokota et al., 2003), and finally an additional Fgf target involved in gastrulation movements has been found more recently (Chung et al., 2005). We suggest that ler2 is a novel example of this growing array of Fgf target genes that have, among other functions, a role in the control of convergent extension, a key process in the establishment of the vertebrate body plan during gastrulation.

\section{Materials and Methods}

\section{Isolation of Xenopus ier2 cDNAs and RT-PCR assay}

Isolation of $\mathrm{X}$. laevis ier2 cDNAs was based on homology searches using the conserved $\mathrm{N}$-terminal region of other vertebrate ier2 genes. Scaffold_649 of the X. tropicalis data base from Ensembl (http://www. ensembl.org/Xenopus_tropicalis) provides full information ier2 genomic sequences. The full-length ORFs of $X$. laevis ier2 was amplified by PCR and subcloned into BamHI-EcoRI sites of the pCS2+ vector. The following primer set was used:

F:5'-AAGATGCAGAGCCGAGCCCAGATG-3',

R:5'-CCCCGGTAGCGCTTAGAAAGCCTC-3'. RT-PCR was performed as previously described (Tanegashima et al., 2004). Total RNA was isolated from $X$. laevis embryos using TRIzol reagent (Invitrogen), and first-strand cDNA synthesis was performed using SuperScript III (Invitrogen). The primers used are:

xier2a F5'- CAAACATTAGCAGGCGAGGGTTC-3', R5'- CATCTTATGTTGTTTCCCTAGTC-3';

xier2b F5'-CAGAGCCGAGCCCAGATG -3', R5'-GGTAGCGCTTAGAAAGCCTC -3'.

Ornithine decarboxylase (ODC) was used as internal control in RT-PCR assays.

\section{Sequence alignment and phylogenetic tree}

Multiple amino acid sequence comparisons and phylogenic tree analysis were carried out with DNASIS MAX version 2.0 (MiraiBio, Hitachi software). Accession numbers are listed in the legend to Fig. 1.

\section{Whole mount in situ hybridization}

Whole mount in situ hybridization was carried out based on Harland (1991). Anti-sense probe was generated by BamHI linearization and transcription with T7 polymerase from pCS2+-Xler2a and b constructs. BM-Purple (Roche) was used for substrates for color reaction.

\section{Injection of mRNA and morpholino}

Capped synthetic xier2 RNA was generated using mMessage mMachine Kit (Ambion) and was injected at levels indicated in the text. Xler2 anti-sense oligonucleotide (MO) targeting the translation initiation sites of $x i e r 2 a, x i e r 2 b$ and standard control MO were purchased from Gene Tools LLC. The sequences are as follows:

Xler2a MO: 5'- CCATCTCACTTTCCAATGCTGAACC-3';

Xler2b MO: 5'- GCATCTTGTGCTGCTTCTTCCGCGC-3'; Ctrl MO 5'-CCTCTTACCTCAGTTACAATTTATA-3'.

The bold letters in Xler2 $\mathrm{MO}$ indicate the translation start sequences. Forty ng of Xler2 $\mathrm{MO}$ or $60 \mathrm{ng}$ of Control MO were injected into the dorsal blastomeres at the four-cell stage.

\section{Animal cap assay}

Animal cap assay were carried out as previously described (Tanegashima et al., 2008).

\section{Acknowledgements}

We thank Martha Rebbert for help with experiments and sequence alignments. This work was supported by the intramural research program of the National Institute for Child Health and Human Development, NIH.

\section{References}

CHARLES, C.H., SIMSKE, J.S., O'BRIEN, T.P. and LAU, L.F. (1990). Pip92: a short-lived, growth factor-inducible protein in BALB/c 3T3 and PC12 cells. Mol Cell Biol 10: 6769-6774.

CHUNG, H.A., HYODO-MIURA, J., NAGAMUNE, T. and UENO, N. (2005). FGF signal regulates gastrulation cell movements and morphology through its target NRH. Dev Biol 282: 95-110.

CONLON, F.L. AND SMITH, J.C. (1999). Interference with brachyury function inhibits convergent extension, causes apoptosis, and reveals separate requirements in the FGF and activin signalling pathways. Dev Biol 213: 85-100.

HARLAND, R.M. (1991). In situ hybridization: an improved whole-mount method for Xenopus embryos. Methods Cell Biol 36: 685-695.

HEISENBERG, C.P., TADA, M., RAUCH, G.J., SAÚDE, L., CONCHA, M.L., GEISLER, R., STEMPLE, D.L., SMITH, J.C. and WILSON, S.W. (2000). Silberblick/Wnt11 mediates convergent extension movements during zebrafish gastrulation. Nature 405: 76-81. 
HONG, S.K. AND DAWID, I.B. (2009). FGF-dependent left-right asymmetry patterning in zebrafish is mediated by ler2 and Fibp1. Proc NatIAcad Sci USA 106:2230-2235.

KELLER, R., DAVIDSON, L., EDLUND, A., ELUL, T., EZIN, M., SHOOK, D. and SKOGLUND, P. (2000). Mechanisms of convergence and extension by cell intercalation. Philos Trans R Soc Lond B Biol Sci 355: 897-922.

LATINKIĆ, B.V. AND LAU, L.F. (1994). Transcriptional activation of the immediate early gene pip92 by serum growth factors requires both Ets and CArG-like elements. J Biol Chem 269: 23163-23170.

NUTT, S.L., DINGWELL, K.S., HOLT, C.E. and AMAYA, E. (2001). Xenopus Sprouty2 inhibits FGF-mediated gastrulation movements but does not affect mesoderm induction and patterning. Genes Dev 15: 1152-1166.

SEPICH, D.S., MYERS, D.C., SHORT, R., TOPCZEWSKI, J., MARLOW, F. and SOLNICA-KREZEL, L. (2000). Role of the zebrafish trilobite locus in gastrulation movements of convergence and extension. Genesis 27: 159-173.

TADA, M. AND SMITH, J.C. (2000). Xwnt11 is a target of Xenopus Brachyury: regulation of gastrulation movements via Dishevelled, but not through the canonical Wnt pathway. Development 127: 2227-2238.
TANEGASHIMA, K., HARAMOTO, Y., YOKOTA, C., TAKAHASHI, S. and ASASHIMA M. (2004). Xantivin suppresses the activity of EGF-CFC genes to regulate nodal signaling. Int J Dev Biol 48: 275-283.

TANEGASHIMA, K., ZHAO, H. and DAWID, I.B. (2008). WGEF activates Rho in the Wnt-PCP pathway and controls convergent extension in Xenopus gastrulation. EMBO J 27: 606-617.

WALLINGFORD, J.B., ROWNING, B.A., VOGELI, K.M., ROTHBÄCHER, U., FRASER, S.E. and HARLAND, R.M. (2000). Dishevelled controls cell polarity during Xenopus gastrulation. Nature 405: 81-85.

WALLINGFORD, J.B., FRASER, S.E. and HARLAND, R.M. (2002).Convergent extension: the molecular control of polarized cell movement during embryonic development. Dev Cell 2: 695-706.

YOKOTA, C., KOFRON, M., ZUCK, M., HOUSTON, D.W., ISAACS, H., ASASHIMA M., WYLIE, C.C. and HEASMAN, J. (2003). A novel role for a nodal-related protein; Xnr3 regulates convergent extension movements via the FGF receptor Development 130: 2199-2212. 


\section{Further Related Reading, published previously in the Int. J. Dev. Biol.}

Establishment of the organizing activity of the lower endodermal half of the dorsal marginal zone is a primary and necessary event for dorsal axis formation in Cynops pyrrhogaster

Koji Sakaguchi, Teruo Kaneda, Miwako Matsumoto, Hiroshi Imoh and Akio S Suzuki

Int. J. Dev. Biol. (2002) 46: 793-800

Dynamin-dependent endocytosis is necessary for convergent-extension movements in Xenopus animal cap explants Oliver Jarrett, Jennifer L Stow, Alpha S Yap and Brian Key

Int. J. Dev. Biol. (2002) 46: 467-473

Regulation of convergent extension in Xenopus by Wnt5a and Frizzled-8 is independent of the canonical Wnt pathway J B Wallingford, K M Vogeli and R M Harland

Int. J. Dev. Biol. (2001) 45: 225-227

Evolution of the organizer and the chordate body plan

J Gerhart

Int. J. Dev. Biol. (2001) 45: 133-153

Mesoderm migration in the Xenopus gastrula

R Winklbauer, M Nagel, A Selchow and S Wacker

Int. J. Dev. Biol. (1996) 40: 305-311

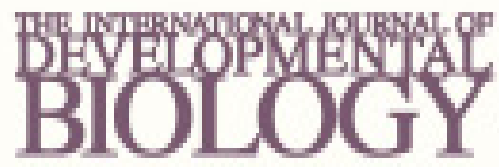

Hohme st Non b/T
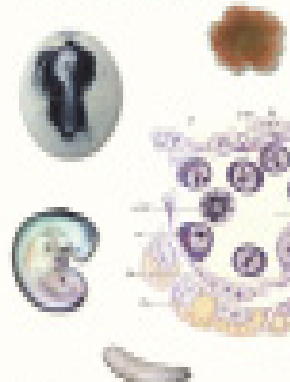

thit.
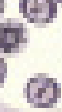

4)

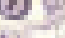

5 yr ISI Impact Factor $(2010)=2.961$
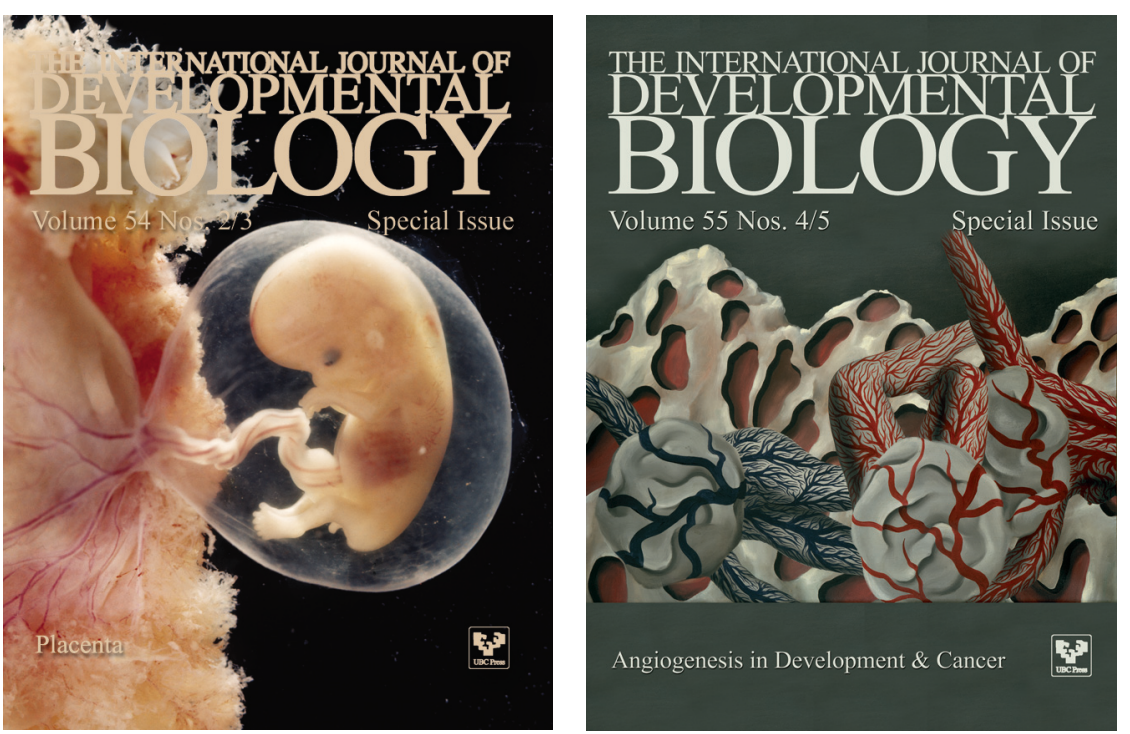